\title{
Long face syndrome: a literature review
}

\begin{abstract}
Long face morphology is a relatively common presentation among orthodontic patients. Despite being described extensively in the orthodontic literature, the long face morphology still remains unclear. The term "long face syndrome" depicts only the vertical component of the three dimensional problem which exists in these patients. Most studies to date concentrate on only the open bite variant of this multifaceted problem. The aim of this article is to comprehensively review the literature and present the varied clinical manifestations, etiology and available treatment modalities of the "Long Face Syndrome".
\end{abstract}

Keywords: morphology, clinical manifestations, hyperdivergent, normodivergent, hypodivergent
Volume 2 Issue 6 - 2015

\author{
Amit Kumar Bansal, Mohit Sharma, Prasanna \\ Kumar, Karan Nehra, Sunil Kumar \\ Department of Dental Surgery,Armed Forces Medical College, \\ India
}

Correspondence: Amit Kumar Bansal, Department of Dental Surgery,Armed Forces Medical College, India,Tel 91 8390551912,Email dr.bansal@rediffmail.com

Received: May 2I, 2015 | Published: September 2, 2015

\section{Introduction}

Long face morphology is a relatively common presentation among orthodontic patients. Classical features include an increased lower facial height, anterior open-bite and a narrow palate. While excessive vertical facial growth can often be recognized clinically, several cephalometric traits are commonly used to classify the underlying vertical skeletal pattern as normal (normodivergent), short (hypodivergent), or long (hyperdivergent). The term "long face syndrome" depicts only the vertical component of the three dimensional problem which exists in these patients.

Both genetic and environmental factors have been associated with the etiology of excessive vertical facial development, although it is likely that more than one subtype of the phenotype exists. Etiological factors such as enlarged adenoids, nasal allergies, weak masticatory muscles, oral habits, and genetic factors have all been implicated in the development of the long face morphology. The treatment objective in a patient having sufficient potential for growth should be to restrain and control maxillary descent and prevent eruption of posterior teeth. When the severity of vertical deformity is so great that reasonable correction cannot be obtained by growth modification or camouflage, the combination of orthodontics and orthognathic surgery may provide the only viable treatment. Despite being described extensively in the orthodontic literature the long face morphology still remains unclear. Most studies concentrate on only the open bite variant of this multifaceted problem. The aim of this article is to comprehensively review the literature and present the varied clinical manifestations, etiology and available treatment modalities of the "Long Face Syndrome".

\section{Literature review}

\section{Nomenclature}

A variety of terms have been used for excessive vertical craniofacial growth, such as the long face syndrome and vertical maxillary excess, ${ }^{1}$ idiopathic long face, ${ }^{2}$ skeletal open-bite, ${ }^{3,4}$ high angle,${ }^{5}$ hyperdivergent, ${ }^{6,7}$ dolichofacial, ${ }^{8}$ and adenoid face. ${ }^{9}$ Although these terms often refer to the same clinical condition, the multiplicity of terms suggests considerable morphological variation within each facial type.

\section{Prevalence}

Two of the largest studies that investigated the prevalence of skeletal facial types were undertaken in the United States, and involved the evaluation of a large orthodontic based patient sample..$^{10,11}$ In both studies, the prevalence of the long face pattern was approximately $22 \%$. This extreme form of vertical craniofacial growth was also reported to be the second most common cause for seeking and receiving orthodontic/surgical treatment. ${ }^{10}$ The prevalence of these vertical growth patterns differed significantly according to Angle's classification of malocclusion, with the highest proportion occurring in the Class III sample (35\%), followed by the Class I (32\%), Class II Division 1 (30\%) and Division 2 (18\%) groups. ${ }^{12}$ These findings were consistent with those of another recent retrospective study investigating the occurrence of skeletal malocclusions in a Brazilian sample. ${ }^{13}$ Recently, Chew ${ }^{14}$ investigated the distribution of dentofacial deformities in an ethnically diverse Asian population receiving orthognathic surgery and found that the overall prevalence of vertical maxillary excess (VME) was nearly $22 \%$, although significant differences existed in the distribution of VME among the three Angle classes. The highest prevalence of VME occurred in the Angle Class I (50\%) and Class II malocclusions (48\%), followed by the Class III group (10\%).

\section{Etiological factors}

Variations in the long face morphology have so far been discussed in terms of skeletal growth imbalances and mandibular rotations, although there still remains a great deal of uncertainty as to what causes or "triggers" these growth patterns..$^{15}$ The multiplicity of growth theories suggests a complex multi factorial etiology that involves genetic, environmental and epigenetic regulation. Several local environmental factors have been implicated in the etiology of the long face morphology; including nasal obstruction, para functional habits and weak muscle activity. ${ }^{16-18}$ Enlarged adenoids and a narrow nasopharynx are common causes of nasal obstruction that can prompt an individual to become a mouth breather. Theoretically, 
the downward and forward tongue position needed for oral respiration may also displace the mandible inferiorly and lead to an increase in vertical dimension. The long face morphology of mouth breathing children may also result from the effects of soft tissue stretching that commonly occur when these individuals overextend their heads to compensate for impaired nasal respiration. ${ }^{16}$ Several authors have found that long face individuals have a narrower nasopharynx than other facial types. ${ }^{16}$ In fact, both anterior and posterior facial heights appear to be positively correlated with all the volumetric measurements of the airway, with the exception of the middle pharyngeal third. ${ }^{19}$

Oral habits such as digit sucking have been associated with the classical traits of the long face morphology. Non-nutritive sucking in the first few years of life is consistently associated with vertical malocclusions such as an anterior open bite. These non-nutritive sucking habits are often not limited to the vertical plane, but may also affect the transverse dimension manifesting as posterior cross-bites. ${ }^{20}$ More recently, Thomas and colleagues used anthropometric points to describe facial morphology, and found a high prevalence of severe facial convexity in adolescents who had been breastfed for relatively short periods and exhibited prolonged mouth-breathing habits that persisted until after the age of 6 years. ${ }^{21}$

\section{Genetic factors}

Different heritability estimates have been reported for various vertical dimensions of the face. For instance, the heritability of total face height is reported to range from 0.8 to 1.3 , while that of the lower anterior face is between 0.9 and 1.6. In contrast, the heritability of the posterior and upper anterior face height ranges from 0.2 to 0.9 and 0.2 to 0.7 , respectively.2. It is noteworthy, however, that heritability studies have a number of limitations that may account for some of the inconsistent findings reported in the literature. Since these estimates are typically derived under different environmental conditions, it is difficult to generalize the findings from one sample to another or even within the same sample over a substantial period of time.

\section{Clinical features (Figure I)}

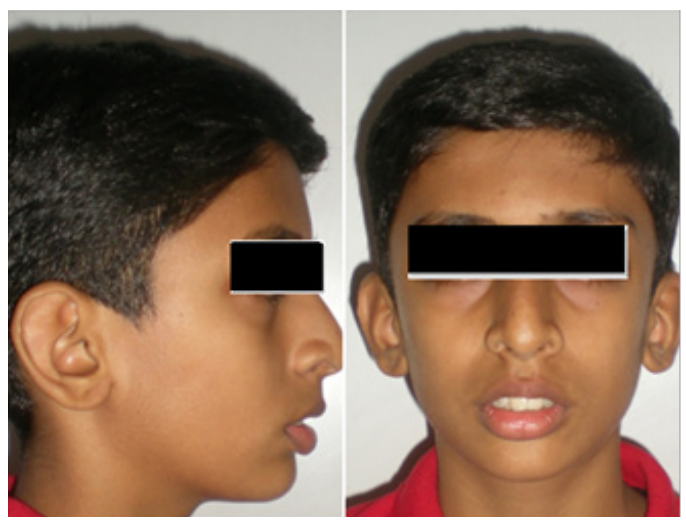

Figure I Clinical features of long face syndrome.

The long face morphology is typically associated with a number of classical features including a longer lower third of the face, facial retrognathism, depressed nasolabial areas, excessive exposure of the maxillary teeth and gingiva, lip incompetence, narrow palate, posterior cross-bites, and an anterior open-bite. ${ }^{1}$ Facial retrognathism, for example, gradually increases with facial divergence and mandibular plane angle..$^{5}$ Other features (such as a dolichocephalic cranium, narrow nasal apertures, small temporal fossa, underdeveloped mandibular processes, narrow and long mandibular symphysis, reduced chin prominence, and large teeth) have also been reported in some individuals with the long face pattern. ${ }^{3}$

Anterior open bites are only found in a limited proportion of individuals with the long face morphology. ${ }^{23}$ Fields and colleagues, ${ }^{24}$ recognized this common misconception and pointed out that "not all long faced patients have open-bites and not all open-bite patients are long faced". The reduced prevalence of anterior open-bites in long face individuals can be attributed to the dentoalveolar compensatory mechanisms, which are capable of masking the underlying skeletal pattern in a large proportion of individuals. ${ }^{25}$

\section{Cephalometric features (Figure 2)}

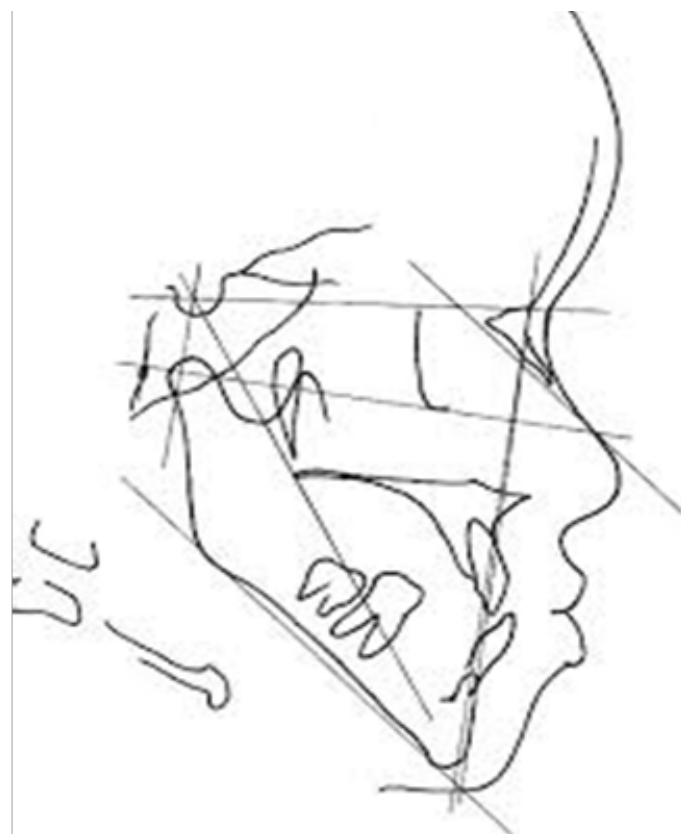

Figure 2 Cephalometric features of long face syndrome.

It is now clear that the majority of the growth disturbances that contribute to the long face morphology occur below the maxillary plane. . $5,9,26,27$ In general, the hyperdivergent pattern results from a combination of dentoalveolar and skeletal features. A number of cephalometric variables that represent these areas have therefore been associated with the long face morphology, including a reduced posterior facial height, greater total facial height, and larger lower anterior facial height, gonial angle, and mandibular plane angle. ${ }^{1,26,28}$ One recent study shows that long faces were predominantly due to increased lower face height..$^{29}$ One of the main limitations of the studies discussed is their confinement to the open-bite variant of the long face morphology.

\section{Morphology and growth patterns}

The relative size of the mandible is significantly smaller in growing children with a hyperdivergent pattern than in those with either the normodivergent or hypodivergent morphologies. ${ }^{30}$ The shape of the mandible is also more variable in those with greater skeletal divergence, and differs from normodivergent individuals at the gonial angle, alveolar process, posterior ramus border, and mandibular plane. Recent studies have shown that the hyperdivergent pattern is associated with thin cortical bone plates which may lead to miniimplant failure especially in maxillary buccal alveolar segments. ${ }^{31}$ 


\section{Treatment}

The clinician must address the three-dimensional dentoalveolar and skeletal problems that present in long face syndrome. Treatment modality depends on the growth potential of the patient when he reports as well as the severity of the dysplasia.

\section{Patients with growth potential}

The primary objective of treatment in a growing child with a long face problem is to restrain and control that area. If vertical movement of the posterior teeth (which is due to a combination of jaw growth and eruption) could be controlled well enough, downward and backward rotation of the mandible could be prevented, and it might even be possible to produce upward and forward rotation of the mandible as growth continues. The long face growth pattern is hard to modify, and it persists until late in the teens; therefore treatment must continue over many years. There has been significant progress in recent years toward effectively controlling and redirecting long face growth, with little or no success in shortening the duration of treatment.

The two traditional methods for impeding excessive vertical growth have been

1. High-pull headgear with maxillary fixed appliance.

2. A functional appliance with bite blocks (Figure 3).

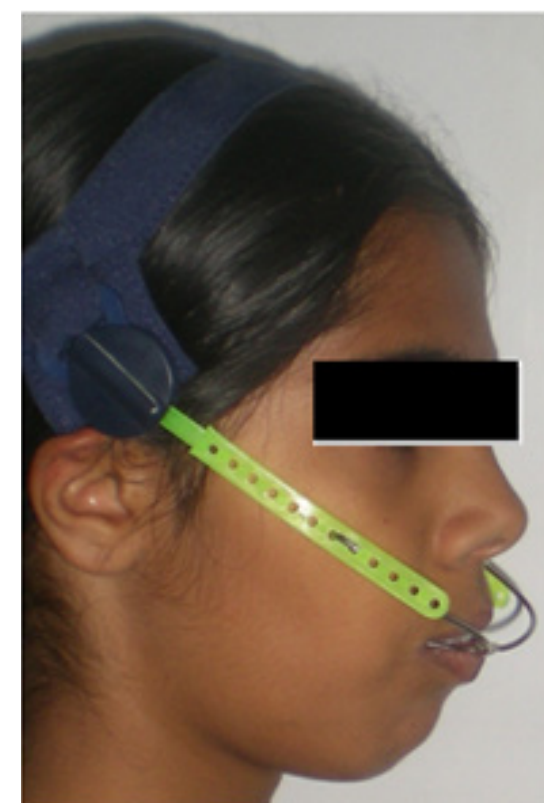

Figure 3 Activator with bite blocks fitted with headgear tubes, so that highpull headgear can be worn while the functional appliance is in use, gives the most effective control of excessive vertical growth. The effect of this appliance on the maxilla is similar to that of a maxillary splint, but it also controls the vertical position of the lower teeth.

\section{Patients with questionable growth potential}

A camouflage treatment plan based on retraction of the upper incisors by extraction of premolars does nothing to help correct the vertical problem. As the upper incisors are retracted they extrude and the nasolabial angle will increase. The fact that vertical growth continues into the late teens can be both a problem and a potential opportunity. A problem exists because the growth pattern tends to further worsen the long face deformity without treatment. An opportunity is present because at least some growth potential usually is present in long face adolescents which can be modified to meet the treatment goals. However, growth modification after the adolescent growth spurt is more a theoretical possibility as it is almost impossible to get adolescents to wear a functional appliance with bite blocks and headgear regularly enough to really control vertical growth. Anterior open bite in adolescents (adults) often can be corrected with orthodontic treatment. Ideally, this would be accomplished by intruding the posterior teeth which is now a possibility with temporary anchorage devices. However, long term stability and the biological limits of safe intrusion which can be achieved are yet to be established..$^{32}$ It has been claimed that a multiloop edgewise appliance, in conjunction with anterior vertical elastics, can produce posterior intrusion and therefore a true correction of the skeletal problem. Even though these results often are stable, recent reports demonstrate what one would expect from the mechanics of the appliance; the open bite correction occurs almost totally by elongating the incisor teeth. ${ }^{14}$ In this borderline situation, a lower border osteotomy of the mandible to bring the chin upward and forward can greatly improve both dental and facial esthetics, because the lower lip relaxes and moves up as the chin is elevated.

\section{Patients with little or no growth potential}

For long face patients with no prospect for successful growth modification, surgery is probably the only treatment option. Orthodontic camouflage does nothing to improve the excessive facial height and can even further worsen it. A patient with a genuine long face problem who does not accept a surgical treatment protocol is better off without any treatment. ${ }^{33}$

\section{Conclusion}

The orthodontic literature widely describes the long face morphology. Various clinical and cephalometric features have been associated with this deformity. It has a multivariate etiology with both genetic and environmental factors associated. Most of the studies concentrate on the open bite variant of the syndrome. In growing individuals, the long face growth pattern is hard to modify, and it persists until late in the teens; therefore treatment with head gear or functional appliances must continue over many years. In individuals in whom growth has seized, surgical approach is the only feasible option.

\section{Acknowledgments}

None

\section{Conflicts of interest}

The author declares that there are no conflicts of interest.

\section{Funding}

None

\section{References}

1. Schendel SA, Eisenfeld J, Bell WH, et al. The long face syndrome: vertical maxillary excess. Am J Orthod. 1976;70(4):398-408. 
2. Willmar K. On Le Fort I osteotomy; A follow-up study of 106 operated patients with maxillo-facial deformity. Scand J Plast Reconstr Surg. 1974; 12(12):1-68.

3. Sassouni V. A classification of skeletal facial types. Am J Orthod. 1969;55(2):109-123.

4. Subtelny JD, Sakuda M. Open-bite: diagnosis and treatment. Am J Orthod. 1964;50(5):337-358.

5. Isaacson JR, Isaacson RJ, Speidel TM, et al. Extreme variation in vertical facial growth and associated variation in skeletal and dental relations. Angle Orthod. 1971;41(3):219-229.

6. Schudy FF. Vertical growth versus anteroposterior growth as related to function and treatment. Angle Orthod. 1964;34(2):75-93.

7. Siriwat PP, Jarabak JR. Malocclusion and facial morphology is there a relationship? An epidemiologic study. Angle Orthod. 1985;55(2):127138.

8. Collett AR, West VC. Terminology of facial morphology in the vertical dimension. Aust Dent J. 1993;38(3):204-209.

9. Quick CA, Gundlach KK. Adenoid facies. Laryngoscope. 1978;88(2):327333.

10. Proffit WR, Phillips C, Dann CT. Who seeks surgical-orthodontic treatment? Int J Adult Orthodon Orthognath Surg. 1990;5(3):153-160.

11. Bailey LJ, Haltiwanger LH, Blakey GH, et al. Who seeks surgicalorthodontic treatment: a current review. Int J Adult Orthodon Orthognath Surg. 2001;16(4):280-292.

12. Willems G, De Bruyne I, Verdonck A, et al. Prevalence of dentofacial characteristics in a Belgian orthodontic population. Clin Oral Investig. 2001;5(4):220-226

13. Boeck EM, Lunardi N, Pinto Ados S, et al. Occurrence of skeletal malocclusions in Brazilian patients with dentofacial deformities. Braz Dent J. 2011;22(4):340-345.

14. Chew MT. Spectrum and management of dentofacial deformities in a multiethnic Asian population. Angle Orthod. 2006;76(5):806-809.

15. Opdebeeck H, Bell WH, Eisenfeld J, et al. Comparative study between the SFS and LFS rotation as a possible morphogenic mechanism. Am J Orthod. 1978;74(5):509-521.

16. Linder-Aronson S. Their effect on mode of breathing and nasal airflow and their relationship to characteristics of the facial skeleton and the dentition. A biometric, rhino-manometric and cephalometro-radiographic study on children with and without adenoids. Acta Otolaryngol. 1970;265:1-132.

17. Tecco S, Caputi S, Tete S, et al. Electromyographic activity of masticatory, neck and trunk muscles of subjects with different mandibular divergence. Across-sectional evaluation. Angle Orthod. 2007;77(2):260-265.

18. Abu Alhaija ES, Al Zo'ubi IA, Al Rousan ME, et al. Maximum occlusal bite forces in Jordanian individuals with different dentofacial vertical skeletal patterns. Eur J Orthod. 2010;32(1):71-77.
19. Ansar J, Maheshwari S, Verma SK, et al. Soft tissue airway dimensions and craniocervical posture in subjects with different growth patterns. Angle Orthod. 2014;85(4):604-610.

20. Cozza P, Baccetti T, Franchi L, et al. Sucking habits and facial hyperdivergency as risk factors for anterior open bite in the mixed dentition. Am J Orthod Dentofacial Orthop. 2005;128(4):517-519.

21. Thomaz EB, Cangussu MC, Assis AM. Maternal breastfeeding, parafunctional oral habits and malocclusion in adolescents: a multivariate analysis. Int J Pediatr Otorhinolaryngol. 2012;76(4):500-506.

22. Amini F, Borzabadi-Farahani A. Heritability of dental and skeletal cephalometric monozygous and dizygous Iranian twins. Orthod Waves. 2009;68(2):72-79.

23. Dung DJ, Smith RJ. Cephalometric and clinical diagnoses of open bite tendency. Am J Orthod Dentofacial Orthop. 1988;94(6):484-490.

24. Fields HW, Proffit WR, Nixon W, et al. Facial pattern differences in long faced children and adults. Am J Orthod. 1984;85(3):217-223.

25. Betzenberger D, Ruf S, Pancherz H. The compensatory mechanism in high-angle malocclusions: a comparison of subjects in the mixed and permanent dentition. Angle Orthod. 1999;69(1):27-32.

26. Nahoum HI. Vertical proportions and the palatal plane in anterior openbite. Am J Orthod. 1971;59(3):273-282.

27. Silva Filho OG, Cardoso GC, Cardoso M, et al. Study of the cephalometric features of Brazilian long face adolescents. Dental Press Journal of Orthodontics. 2010;15(4):35.e1-35.e12.

28. Cangialosi TJ. Skeletal morphologic features of anterior open bite. Am J Orthod. 1984;85(1):28-36.

29. Yale H, Park YS, Lee SP. Do long-faced subjects really have a long anterior face? A longitudinal study. Am J Orthod Dentofacial Orthop. 2014;145(6):799-806

30. Ferrario VF, Sforza C, De Franco DJ. Mandibular shape and skeletal divergency. Eur J Orthod. 1999;21(2):145-153.

31. Ozdemir F, Tozlu M, Germec-Cakana D. Cortical bone thickness of the alveolar process measured with cone-beam computed tomography in patients with different facial types. Am J Orthod Dentofacial Orthop. 2013;143(2):190-196.

32. Scheffler NR, Proffit WR, Phillips C. Outcomes and stability in patient with anterior open bite and long anterior face height treated with temporary anchorage devices and a maxillary intrusion splint. $\mathrm{Am} \mathrm{J}$ Orthod Dentofacial Orthop. 2014;146(5):594-602.

33. Proffit WR, White RP Jr, Sarver DM. Contemporary treatment of Dentofacial Deformity, Mosby, USA: 2003;464-506. 